\title{
Revealing the Jet Structure of GRB 030329 with High Resolution Multicolor Photometry
}

\author{
J. Gorosabel ${ }^{1}$, A.J. Castro-Tirado ${ }^{1}$, E. Ramirez-Ruiz ${ }^{2,3}$, J. Granot ${ }^{4}$, N. Caon ${ }^{5}$, L.M. \\ Cairós $^{5}$, E. Rubio-Herrera ${ }^{6}$, S. Guziy ${ }^{1}$, A. de Ugarte Postigo ${ }^{1}$, M. Jelínek ${ }^{1}$
}

\begin{abstract}
We present multicolor optical observations of the nearby $(z=0.1685)$ GRB 030329 obtained with the same instrumentation over a time period of 6 hours for a total of an unprecedented 475 quasi-simultaneous $B V R$ observations. The achromatic steepening in the optical, which occurs at $t \sim 0.7$ days, provides evidence for a dynamic transition of the source, and can be most readily explained by models in which the GRB ejecta are collimated into a jet. Since the current state-of-the-art modeling of GRB jets is still flawed with uncertainties, we use these data to critically assess some classes of models that have been proposed in the literature. The data, especially the smooth decline rate seen in the optical afterglow, are consistent with a model in which GRB 030329 was a homogeneous, sharp-edged jet, viewed near its edge interacting with a uniform external medium, or viewed near its symmetry axis with a stratified wind-like external environment. The lack of short timescale fluctuations in the optical afterglow flux down to the 0.5 per cent level puts stringent constraints on possible small scale angular inhomogeneities within the jet or fluctuations in the external density.
\end{abstract}

Subject headings: gamma rays: bursts — shock waves — ISM: jets and outflows

\footnotetext{
${ }^{1}$ Instituto de Astrofísica de Andalucía (CSIC), 18008 Granada, Spain

${ }^{2}$ Institute for Advanced Study, Einstein Drive, Princeton NJ 08540, USA

${ }^{3}$ Chandra Fellow

${ }^{4}$ KIPAC, Stanford University, Stanford CA 94309, USA

${ }^{5}$ Instituto de Astrofísica de Canarias, 38205 La Laguna, Tenerife, Spain

${ }^{6}$ Astronomical Inst. Anton Pannekoek, 1098 SJ Amsterdam, Netherlands
} 


\section{Introduction}

A watershed event occurred on 29 March 2003 when HETE-II localized rapidly a long GRB (Vanderspeck et al. 2003). The prompt discovery of the fading optical counterpart (Torii et al. 2003; Price et al. 2003; Uemura et al. 2004; Sato et al. 2003) combined with its exceptional brightness allowed densely sampled observations of this afterglow (Lipkin et al. 2004 and references therein). At $z=0.1685$ (Greiner et al. 2003a), GRB 030329 is the third closest GRB to date for which an optical afterglow (OA) has been discovered. It is by detailed study of such nearby events that we have learned the most about the range of physical processes relevant to GRBs. GRB 030329 was the first burst with a secure spectroscopic association to a type Ic supernova (Stanek et al. 2003; Hjorth et al. 2003; Sokolov et al. 2003) and the monitoring of its afterglow polarization is the best to date (Greiner et al. 2003b). In its early evolution, the GRB 030329 afterglow also provided strong evidence for slow shells with modest Lorentz factors carrying most of the kinetic energy in the relativistic ejecta (Granot et al. 2003).

Follow-up observations within $1.5 \mathrm{hr}$ (Peterson \& Price 2003) discovered an OA with $R \sim 12$, brighter than any OA previously detected at a similar epoch. Soon after, intensive spectroscopic monitoring revealed a supernova, SN 2003dh, with a spectrum very similar to SN 1998bw. These observations showed that $\sim 0.4 M_{\odot}$ of ${ }^{56} \mathrm{Ni}$, the parent nucleus of ${ }^{56} \mathrm{Co}$, was formed in the explosion, suggesting a progenitor main sequence mass of 25-40 $M_{\odot}$ (Mazzali et al. 2003). The GRB 030329 afterglow was monitored in the radio, optical, and X-ray. The radio image was resolved by the VLBA (Taylor et al. 2004), its diameter measured to be $\sim 0.2 \mathrm{pc}(\sim 0.5 \mathrm{pc})$ after 25 days (83 days), indicating an average apparent expansion velocity of $\sim 5.7 c(\sim 4.1 c)$. This decelerating apparent superluminal expansion agrees with expectations of the standard afterglow theory (Oren et al. 2004). The image size did not change much between 83 and 217 days (Taylor et al. 2005), favoring a uniform external medium and a jet with little lateral spreading. The GRB 030329 lightcurve indeed showed a pan-chromatic steepening at about half a day, which has been attributed to a jet-like outflow (Price et al. 2003).

GRB 030329 belongs to a growing group of GRBs for which densely monitored OA lightcurves show significant deviations relative to a smooth power-law decay - most notably GRB 021004 (Bersier et al. 2003; de Ugarte Postigo et al. 2005) and GRB 011211 (Jakobsson et al. 2004). These deviations are interesting for constraining the activity and identity of the central engine driving the GRB, but complicate the simple jet interpretation mentioned above. In this Letter, we report observations of the GRB 030329 OA that overcome the previous sampling limitations with a total of an unprecedented 475 high resolution, quasisimultaneous $B V R$-band observations obtained with the same instrumentation over a time 
period of 6 hours. Here, for the first time, we establish a detailed multicolor optical lightcurve around the break time and argue that in concert they provide good support for the jet interpretation of the lightcurve break. The paper is structured as follows. $\S 2$ details the observations and data analysis, $\S 3$ discusses the physical implications, and our conclusions are summarized in $\S 4$.

\section{Observations}

We commenced observations of the GRB 030329 OA, $9.44 \mathrm{hr}$ after the GRB, using the ALFOSC camera at the $2.5 \mathrm{~m}$ Nordic Optical Telescope. We continued monitoring with the same instrumentation over a time period of $6 \mathrm{hr}$. The data were taken in sequential cycles of $B V R$-band images. The monitoring is composed of 157 images in $B, 158$ in $V$, and 160 in the $R$-band. In order to enhance time resolution, the $2048 \times 2048$ pixel chip of ALFOSC was binned by $2 \times 2$ and trimmed to a $800 \times 800$ pixel window. The typical exposure time per frame was $20 \mathrm{~s}$, achieving a time resolution (including readout time) of about $\sim 150 \mathrm{~s}$ between two consecutive images taken in the same band. The photometry is based on aperture photometry running under IRAF.

The field of view center was selected to include the OA and four reference stars (A, $\mathrm{B}, \mathrm{C}$ and $\mathrm{D}$, see Fig. 1). The OA magnitude $(R \sim 15)$ is similar to those of the reference stars (Henden 2003), showing photometric errors (typically $<1 \%$ ) comparable to the OA. Although the airmass during our observations did not reach extreme values $(S e c \mathrm{z}<1.6)$, we introduced airmass-dependent, color term corrections to derive the OA $B V R$-band magnitudes. This correction is more prominent at the $B$-band, reaching an OA flux increment up to $0.007 \mathrm{mag}$ at $S e c \mathrm{z}=1.6$. For calibration, at a given aperture, four different $\mathrm{OA}$ lightcurves were obtained per filter, each one based on a different reference star. We averaged the residuals of the four lightcurves, instead of averaging over the individual, calibrated lightcurves, so that the error of the residuals was not affected by the zero point uncertainty of the reference stars. This allowed to decrease the potential dispersion contribution due to the reference stars' statistical fluctuations. This method provided for a given photometric aperture the mean $B V R$-band fluctuations. As a sanity check, the process was repeated for 6 apertures, ranging from 1 to 2.5 times the stars' FWHM. The final lightcurve and the shape of the associated residuals (see bottom panel of Fig. 2) were independent of the

adopted aperture. The final magnitudes were obtained averaging the lightcurves yielded for the 6 apertures (see Table 1).

The $B, V$ and $R$-band lightcurves, along with numerous points from other groups reported in the literature, are plotted in Fig. 2, where it is evident that the lightcurve 


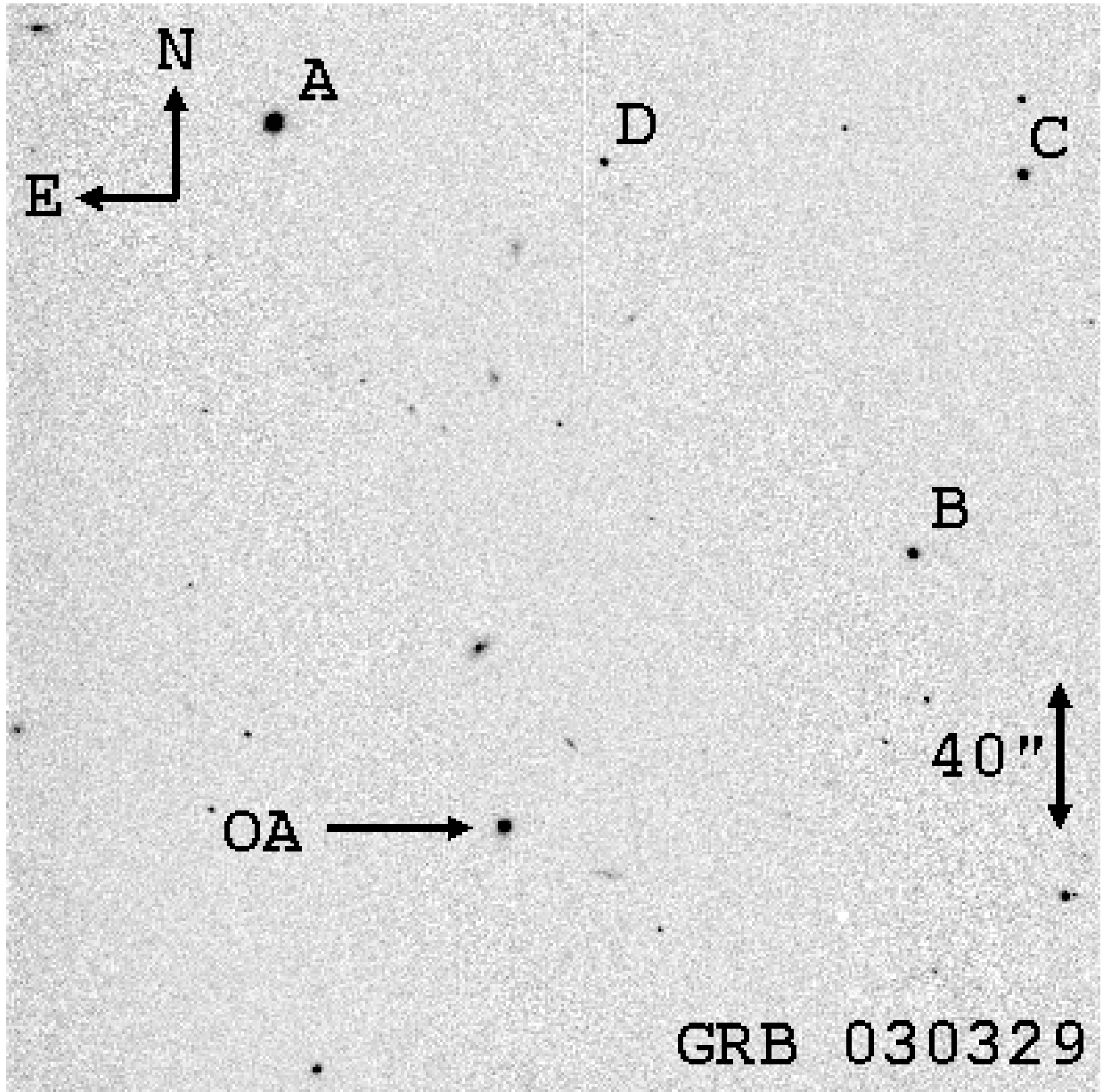

Fig. 1. - B-band image of the field centered on the optical afterglow of GRB 030329. The reference stars A, B, C, D and the OA are indicated. 
steepens contemporaneously in all bands, between $\sim 0.3$ days and $\sim 0.9$ days. In order to characterize the shape of the afterglow lightcurve near the jet break time, $t_{j}$, we fit the data with the phenomenological Beuermann et al. (1999) function (Eq. 1$) ; F(t, \nu)=$ $F_{0} \nu^{\beta}\left[\left(t / t_{j}\right)^{-\alpha_{1} s}+\left(t / t_{j}\right)^{-\alpha_{2} s}\right]^{-1 / s}+F_{h o s t}(\nu)$, which provides a good description of the data, featuring a smooth transition (whose sharpness is parametrized by $s$ ) between the asymptotic power-law indexes $\alpha_{1}$ and $\alpha_{2}$ at early and late times, respectively. $F_{\text {host }}(\nu)$ corresponds to the $B V R$-band host galaxy flux, which was fixed using the magnitudes reported by Gorosabel et al. (2005). Fitting the $B, V$, and $R$-band data simultaneously yields $t_{j}=0.72 \pm 0.2$ days, $s=1.6 \pm 0.7, \beta=-0.89 \pm 0.15, \alpha_{1}=-0.88 \pm 0.1$, and $\alpha_{2}=-2.52 \pm 0.3$, where the uncertainties are formal $1 \sigma$ errors. ${ }^{1}$ The $\chi^{2}$ for the fit is acceptable: 446 for 638 degrees of freedom. The lightcurve shows no significant short timescale variability on top of the smooth trend described by the functional fit above, down to $\Delta B \sim 0.005, \Delta V \sim 0.005$, and $\Delta R \sim 0.005$ (i.e. $\Delta F / F \lesssim 0.5 \%)$ on timescales $\gtrsim 150 \mathrm{~s}\left(\Delta t / t \gtrsim 10^{-2.5}\right)$. The low $R$-band dispersion agrees with that found by Urata et al. (2004). A linear fit to $\beta$ vs. $\log \left(t-t_{0}\right)$ yields a slope of $-0.037 \pm 0.028$, hence consistent with no spectral evolution between $9.44 \mathrm{hr}$ and $15.56 \mathrm{hr}$ after the burst.

\section{Discussion}

\subsection{Evidence for a jet in GRB 030329}

A pan-chromatic steepening in the OA flux decay, like the one reported here in three optical bands ( $B V R$ ) for GRB 030329, was predicted for an outflow collimated into a narrow jet (Rhoads 1999). Such a "jet break" occurs when the Lorentz factor, $\Gamma$, of the shocked external medium drops below $\theta_{0}^{-1}$, where $\theta_{0}$ is the initial half-opening angle of the jet (Sari, Piran \& Halpern 1999). At this stage the edge of the jet becomes visible. In principle, lateral expansion of the jet might also become important when $\Gamma \theta_{0} \sim 1$, as the edge of the jet and its center come into causal contact. However, hydrodynamic simulations (Granot et al. 2001) show a modest degree of lateral spreading as long as the jet is relativistic, suggesting that the jet break in the lightcurve is caused primarily because the edge of the jet becomes visible.

At early times, $t \ll t_{j}$, the lightcurve is given by the spherical solution, where in the optical (depending on the density profile assumed for the external matter) $\alpha_{1}=3(1-p) / 4$ for a uniform density profile and $\alpha_{1}=(1-3 p) / 4$ for a wind. The closure relation $\alpha_{1}-3 \beta / 2=0$

\footnotetext{
${ }^{1}$ Including measurements by other groups (see Fig. 2) before and after our observations result in a slightly sharper break, $s=2.3 \pm 0.3$.
} 

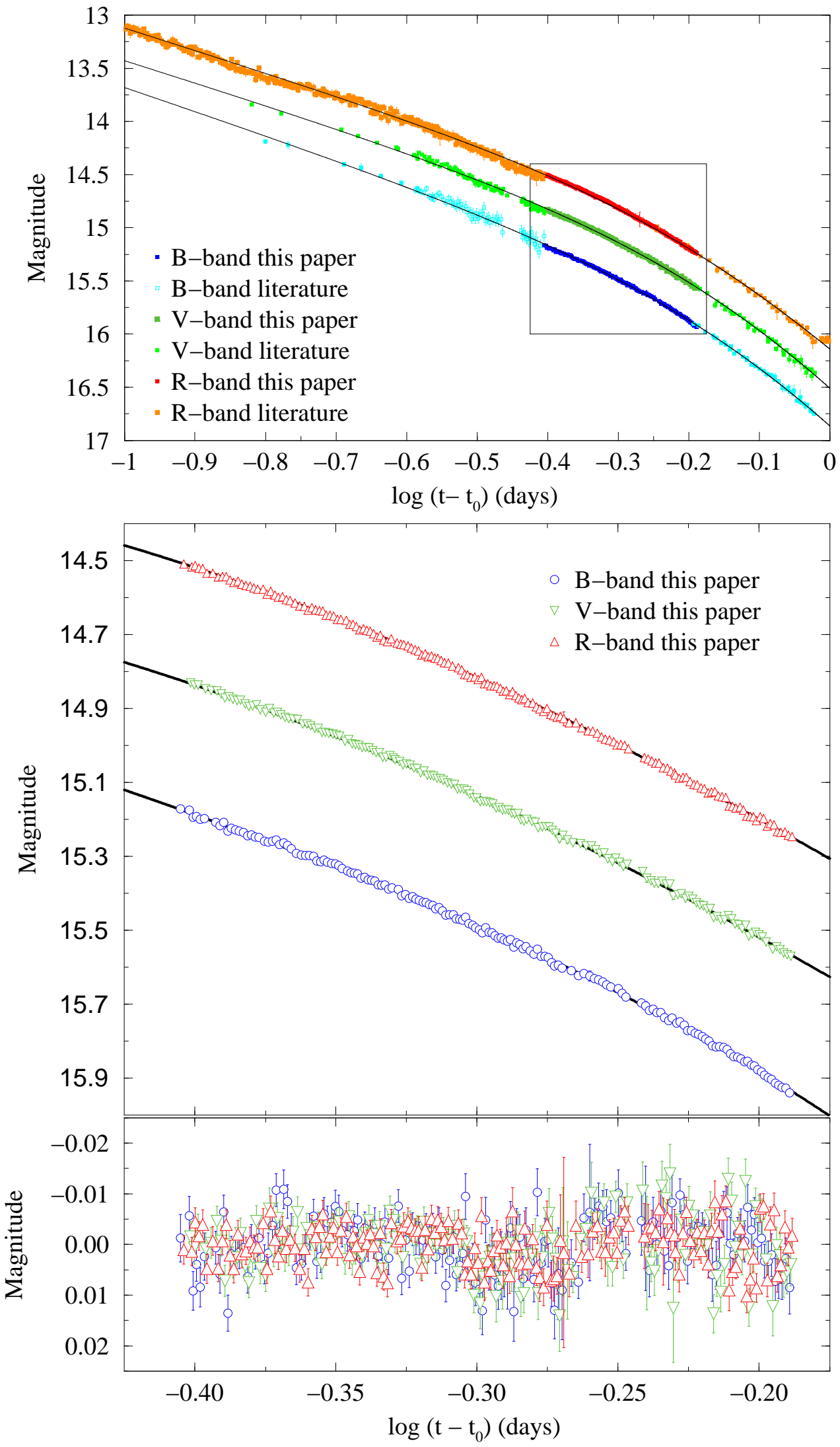

Fig. 2.- $B V R$-band lightcurves of the GRB 030329 OA. In addition to photometry from our group (middle panel), we have augmented the lightcurves with data from the literature 
$(1 / 2)$ is expected for a uniform density (wind). Our observed value, $\alpha_{1}-3 \beta / 2=-0.45 \pm 0.3$, favors a uniform density, for which $p=1+4 \alpha_{1} / 3=2.2 \pm 0.2 p=1+2 \beta=2.78 \pm 0.3$ are marginally consistent. For relativistic lateral spreading of the jet in its own rest frame, $\alpha_{2}=-p$ (Sari, Piran \& Halpern 1999). For little or no lateral spreading, $\alpha_{1}-\alpha_{2}=$ $-2(d \log \Gamma / d \log t)=(3-k) /(4-k)$ for a power law external density, $\rho_{\text {ext }} \propto r^{-k}$, so that $\alpha_{2}=-3 p / 4$ for $k=0$. However, the value of $\alpha$ immediately after the break is smaller than its asymptotic value ${ }^{2}$, underestimating $\alpha_{2}$. Therefore, our measured value of $\alpha_{2}=-2.52 \pm 0.3$ is consistent with this picture. For $t_{j}=0.72 \pm 0.2$ days, we find $\theta_{0}=0.097\left(E_{51} / n_{0}\right)^{-1 / 6}$ $\left[\theta_{0}=0.13\left(E_{51} / A_{*}\right)^{-1 / 2}\right]$ for $k=0(k=2)$. Using the constraints on $E_{51} / n_{0}\left(E_{51} / A_{*}\right)$ from the radio image size (Granot et al. 2005), we derive ${ }^{3}, \theta_{0} \sim 0.083-0.14(\sim 0.27-0.55)$.

The densely monitored observations presented here provide a unique opportunity to critically assess different jet models that have been proposed, and to constrain the jet structure and the external density profile. We start with a uniform external medium, and consider two models of increasing complexity. First, we consider a simple semi-analytical model (model 1 of Granot \& Kumar 2003; Ramirez-Ruiz et al. 2005), which neglects lateral spreading. This approximation is consistent with results of hydrodynamic simulations (Cannizzo et al. 2003), which are also considered here. The advantage of the hydrodynamic model is a reliable and rigorous treatment of the jet dynamics, which provides insight on the behavior of the jet and lightcurves. We use Eq. 1 to determine the sharpness parameter, $s$, of the jet break. While the prompt GRB seen by all observers within the initial jet aperture, $\theta_{\text {obs }}<\theta_{0}$, is the same for a uniform jet, the jet break is sharper at smaller $\theta_{\text {obs }}$ (Granot et al. 2001). For a uniform jet, we find $s=7.3,4.4,1.9,1.1,1.6$ for $\theta_{\text {obs }}=\left[0, \frac{1}{4}, \frac{1}{2}, \frac{3}{4}, 1\right] \theta_{0}$. While the basic lightcurve features for $\theta_{\text {obs }}<\theta_{0}$ are similar in both models, the hydrodynamic model gives sharper breaks; e.g., we find $s$ values ranging from ${ }^{4} s\left(\theta_{\text {obs }}=0\right) \sim 10$ to $s\left(\theta_{\text {obs }}=\theta_{0}\right) \sim 1$.

For a wind external density profile $(k=2)$, the jet break is smoother, because both the Lorentz factor decreases more slowly with time (Kumar \& Panaitescu 2000) and the limb-brightening of the afterglow image is weaker (Granot \& Loeb 2001) when compared to a uniform external medium $(k=0)$. For the uniform jet model, we find $s=2.2,1.8,1.2,0.8,1.1$ for $\theta_{\mathrm{obs}}=\left[0, \frac{1}{4}, \frac{1}{2}, \frac{3}{4}, 1\right] \theta_{0}$. While the slow change in image size observed between 83 and

\footnotetext{
${ }^{2}$ This has been shown in numerical and semi-analytic (Rossi et al. 2004) studies, and occurs because as the edge of the jet becomes visible, we first start "missing" the outer part of the afterglow image, which is limb brightened (Granot et al. 1999).

${ }^{3}$ We take into account an increase in energy by a factor of $\sim 10$ due to refreshed shocks that occurred between $t_{j}$ and the image size measurements.

${ }^{4}$ The values given here are for $p=2.2$, which is appropriate for GRB 030329. Generally, we expect a sharper jet break (larger $s$ ) for large values of $p$ (Rossi et al. 2004).
} 
217 days favors a uniform external medium (Taylor et al. 2005), the sharpness change does not. The relatively smooth break in GRB 030329, with $s=1.6 \pm 0.7$, suggests $\theta_{\text {obs }} / \theta_{0} \sim 0.7-1$ $(\lesssim 0.7)$ for $k=0(k=2)$. However, a large fraction of OAs (Zeh, Klose \& Kann 2005) show evidence for sharper breaks, with $s$ values consistent with lower $\theta_{\text {obs }} / \theta_{0}$ values for $k=0$, but are too sharp for any viewing angle for $k=2$.

\subsection{GRB 030329 and its variability}

GRB 030329 belongs to a growing group of bursts for which densely monitored lightcurves show significant "bumps" and "wiggles" relative to a simple power-law decay. Fig. 3 shows the temporal evolution of the variability timescale, $\Delta t / t$, of the various wiggles observed in the GRB 030329 lightcurve together with other reported variations from a simple power-law decay in X-ray and GRB OAs. The short-term wave-like behavior of the GRB 030329 optical lightcurve is unprecedented. The early OA wiggle seen at $t \sim 10^{4} \mathrm{~s}$ (Sato et al. 2003) could be interpreted as the result of the shock wave encountering an external medium of variable density (e.g. Wang \& Loeb 2000; Ramirez-Ruiz et al. 2001; Lazzati et al. 2002). The pronounced bumps between a day and a week, however, are most readily explained by refreshed shocks, i.e. slower shells that were ejected from the source near the end of the GRB and catch up with the afterglow shock at a later time, when the latter decelerates to slightly below the shells' Lorentz factor (Granot et al. 2003). These shells collide with the shocked fluid behind the afterglow shock and increase its energy (Rees \& Mészáros 1998; Panaitescu et al. 1998; Nakar et al. 2003; Heyl \& Perna 2003).

Fig. 3 shows that while the OA shows substantial variability at $t \gtrsim 10^{5}$ s after the burst, we can set a very strict upper limit on the variability shortly before the onset of the wave-like behavior of the optical lightcurve, of $\Delta F / F \lesssim 5 \times 10^{-3}$ on timescales $10^{-2.5} \lesssim \Delta t / t \lesssim 0.25$. The low level of variability before one day constrains both the possible angular inhomogeneity within the afterglow shock and density variations in the external medium. Let us consider variations in the energy per solid angle $E_{\text {iso }}$ (external density $n$ ) on a typical angular (length) scale $\theta_{E}(l)$ which cause a fractional change $\Delta F_{0} / F_{0}$ in the local emission from each such small region. ${ }^{5}$ The total number of regions contributing to the total observed flux is $N \sim$ $\left(\Gamma \theta_{E}\right)^{-2}\left(N \sim \Gamma^{-2}(R / l)^{3}\right)$ and since around the jet break time $\Gamma \sim \theta_{0}^{-1}$ then $N \sim\left(\theta_{0} / \theta_{E}\right)^{2}$ $\left(N \sim \theta_{0}^{2}(R / l)^{3}\right)$. We expect the fluctuations in the total observed flux on a timescale $\Delta t$ to be of the order $\Delta F / F \sim N^{-1 / 2}(\Delta t / t)^{1 / 2}\left(\Delta F_{0} / F_{0}\right)$. For our limits this implies $N^{1 / 2} \gtrsim$

\footnotetext{
${ }^{5}$ Since in our case $F_{\nu} \propto n^{1 / 2} E_{\text {iso }}^{(3+p) / 4}$, a larger fractional change in the density is needed in order to produce the same variation in flux.
} 


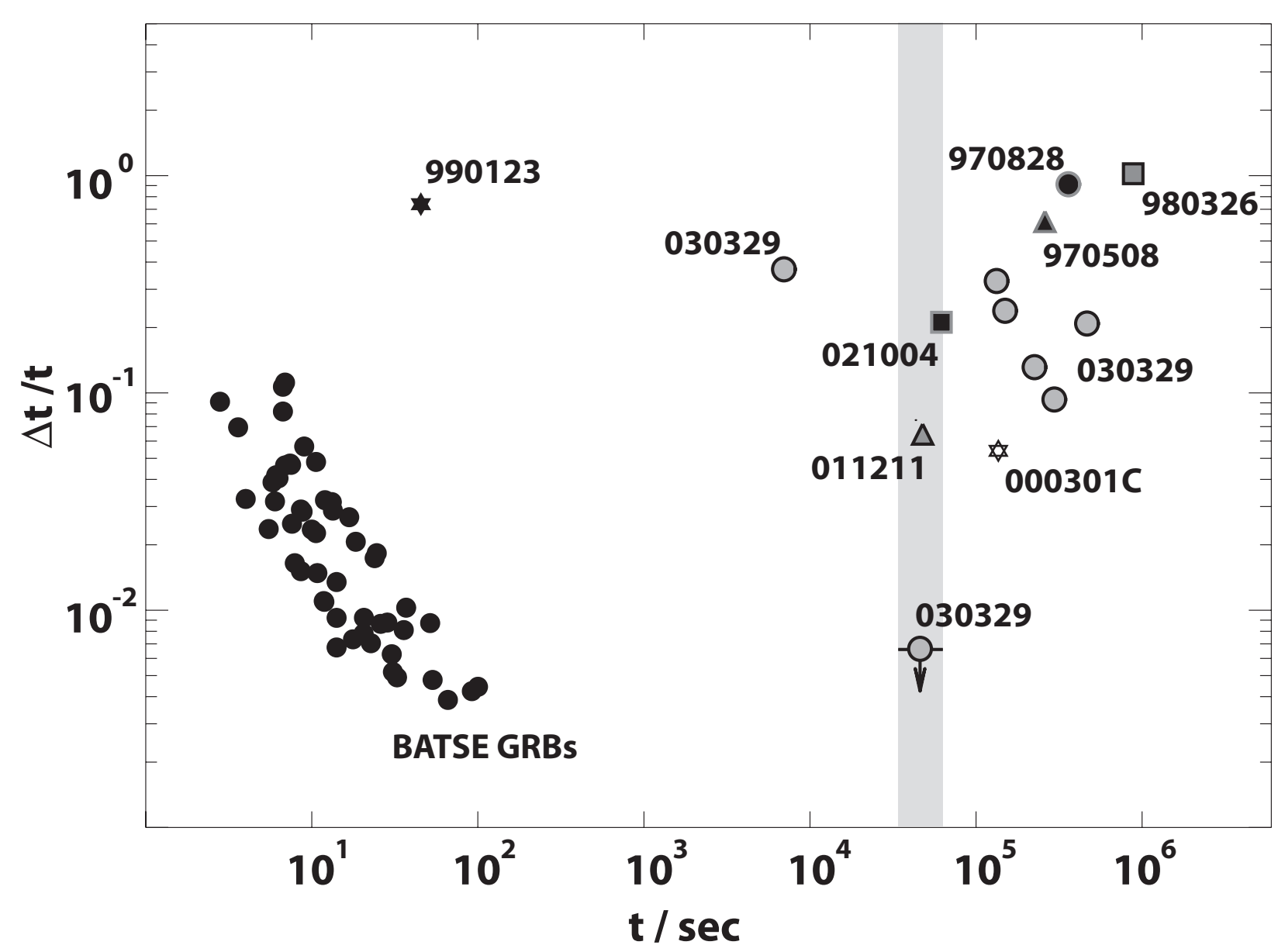

Fig. 3.- Evolution of the variability, $\Delta t / t$, of the GRB 030329 OA (grey circle at $t \lesssim 10^{4} \mathrm{~s}$, Sato et al. (2003); circle in shaded region, this paper; grey circles at $t>10^{5} \mathrm{~s}$, Lipkin et al. (2004)). Reported variations from a simple power-law decay in the X-ray afterglows of GRB 970508 (Piro et al. 1998) and 970828 (Yoshida et al. 1998); and in the OAs of GRB 980326 (Bloom et al. 1999), GRB 990123 (Akerlof et al. 1999), GRB 011211 (Jakobsson et al. 2004), GRB 021004 (Bersier et al. 2003), and GRB 000301C (Garnavich et al. 2000) are also illustrated. The solid symbols are long, complex BATSE GRBs for which $\Delta t$ has been estimated from the width of the autocorrelation function (Fenimore et al. 1999), and $t$ is the GRB duration. This figure demonstrates that while the GRB 030329 OA displays substantial variability at $t \gtrsim 10^{5} \mathrm{~s}$ after the burst, we observe (shaded region) no significantly variability, $\Delta t / t \lesssim 10^{-2.5}$, shortly before the onset of the wave-like behavior. 
$100\left(\Delta F_{0} / F_{0}\right)$, i.e. $l / R \lesssim 10^{-2}\left(\theta_{0} / 0.1\right)^{2 / 3}\left(\Delta F_{0} / F_{0}\right)^{-2 / 3}$ where $R\left(t_{j}\right) \approx 5.4 \times 10^{17}\left(E_{50} / n_{0}\right) \mathrm{cm}$ or $\theta_{E} \lesssim 10^{-3}\left(\theta_{0} / 0.1\right)\left(\Delta F_{0} / F_{0}\right)^{-1}$.

\section{Conclusions}

We report on high-resolution photometric observations of the GRB 030329 OA obtained with the same instrumentation over a time period of 6 hours for a total of 475 quasisimultaneous $B V R$-band measurements with typical statistical photometric errors below 0.005 mag. Thus our data, to our knowledge, constitute the most complete and dense sampling of the jet like behavior of a GRB afterglow to date. Our well-sampled multicolor lightcurve is well described by a physical model where the ejecta are collimated in a jet. We conclude that the observations, especially the smooth decline rate seen in the OA, are consistent with a model in which GRB 030329 was a sharp-edged GRB jet. We consider both a uniform and a wind density profile. While both the observed evolution of the image size and the relation between the observed spectral and temporal indexes favor a uniform medium, both models are consistent with smooth jet break in our data. Moreover, we derive strict upper limits on the afterglow short time scale variability during our observations, of $\lesssim 0.5 \%$, which puts strong limits on possible fluctuations in the energy per solid angle within the jet and in the external density. Evolving photometric properties provide a unique diagnostic tool for GRB studies, and the complex lightcurve of the GRB 030329 OA emphasizes that data should be acquired with high sampling frequency.

This work is supported by IAS/NASA through a Chandra Fellowship award PF3-40028 (ERR) under contract DE-AC03-76SF00515 (JG), and by the Spanish Ministry of Science through programs ESP2002-04124-C03-01 and AYA2004-01515.

\section{REFERENCES}

Akerlof, C., et al. 1999, Nature,398, 400

Bersier, D., et al. 2003, ApJ, 584, L43

Beuermann, K., et al. 1999, A\&A, 352, L26.

Bloom, J. S., et al. 1999, Nature, 401, 453

Cannizzo, J. K., Gehrels, N., \& Vishniac, E. T. 2004, ApJ, 601, 380 
de Ugarte Postigo, et al. 2005, A\&A, 443, 841

Fenimore, E. E., et al. 1999, ApJ, 512, 683

Garnavich, R. M., Loeb, A., \& Stanek, K. Z. 2000, ApJ, 544, L11

Gorosabel, J., et al. 2005, A\&A, 444, 711

Granot, J., Piran, T., \& Sari, R. 1999, ApJ, 513, 679

Granot, J., et al. 2001, in Gamma-Ray Bursts in the Afterglow Era, ed. E. Costa, F. Frontera, \& J. Hjorth (Berlin: Springer), 312

Granot, J., \& Loeb, A. 2001, ApJ, 551, L63

Granot, J., Nakar, E., \& Piran, T. 2003, Nature, 426, 138

Granot, J., \& Kumar, P. 2003, ApJ, 591, 1086

Granot, J., Ramirez-Ruiz, E., \& Loeb, A. 2005, ApJ, 618, 413

Greiner, J., et al. 2003a, GRB Circ., 2020, 1

Greiner, J., et al. 2003b, Nature, 426, 157

Heyl, J. S., \& Perna, R. 2003, ApJ, 586, L13

Henden, A., 2003, GCN Circ., 2082.

Hjorth, J., et al. 2003, Nature, 423, 847

Jakobsson, P., et al. 2004, NewA, 9, 435

Kumar, P., \& Panaitescu, A. 2000, ApJ, 541, L9

Lazzati, D., Rossi, E., Covino, S., Ghisellini, G., \& Malesani, D. 2002, A\&A, 396, L5

Lipkin, Y. M. et al. 2004, ApJ, 606, 381

Mazzali, P. A., et al. 2003, ApJ, 599, L95

Nakar, E., Piran, T., \& Granot, J. 2003, NewA, 8, 495

Oren, Y., Nakar, E., \& Piran, T. 2004, MNRAS, 353, L35

Panaitescu, A., Mészáros, P., \& Rees, M. J. 1998, ApJ, 503, 314 
Peterson, B.A., \& Price, P., 2003, GCN Circ. 1985

Piro, L., et al. 1998, A\&A, 331,L41

Price, P. A., et al. 2003, Nature, 423, 844

Ramirez-Ruiz, E., Dray, L. M., Madau, P., \& Tout, C. A. 2001, MNRAS, 327, 829

Ramirez-Ruiz, E., Granot, J., Kouveliotou, C., Woosley, S. E., Patel, S. K., \& Mazzali, P. A. 2005, ApJ, 625, L91

Rees, M. J., \& Mészáros, P. 1998, ApJ, 496, L1

Rhoads, J. E. 1999, ApJ, 525, 737

Rossi, E., Lazzati, D., Salmonson, J. D., \& Ghisellini, G. 2004, MNRAS, 354, 86

Sari, R., Piran, T., \& Halpern, J. P. 1999, ApJ, 519, L17

Sato, R., et al. 2003, ApJ, 599, L9

Sokolov, K. Z., et al. 2003, BSAO 56, 5

Stanek, K. Z., et al. 2003, ApJ, 591, L17

Taylor, G. B., Frail, D. A., Berger, E., \& Kulkarni, S. R. 2004, ApJ, 609, L1

Taylor, G. B., et al. 2005, ApJ, 622, 986

Torii, K., et al. 2003, ApJ, 597, L101

Uemura, M., et al. 2004, PASJ 56,77

Urata, et al. 2004, ApJL 601, L17

Vanderspeck, R. et al. 2003, GCN Circ. 1997.

Wang, X., \& Loeb, A. 2000, ApJ, 535, 788

Yoshida, A., et al. 1998, in Gamma-Ray Bursts : 4th Huntsville, ed. C. A. Meegan, R. D. Preece, \& T. M. Koshut. (New York: AIP 428), 441

Zeh, A., Klose, S., \& Kann, D. A. 2005, ApJ, in press (astro-ph/0509299) 
Table 1. To be published as an electronic table.

\begin{tabular}{ccc}
\hline \hline MJD & Band & Magnitude \\
\hline $2728.3776157-2728.3777315$ & B & $15.166 \pm 0.014$ \\
$2728.3787153-2728.3788310$ & R & $14.502 \pm 0.023$ \\
$2728.3803472-2728.3805208$ & B & $15.170 \pm 0.013$ \\
$2728.3807870-2728.3809028$ & V & $14.828 \pm 0.009$ \\
$2728.3811690-2728.3812847$ & R & $14.509 \pm 0.023$ \\
$2728.3815394-2728.3817130$ & B & $15.188 \pm 0.013$ \\
$2728.3819792-2728.3820949$ & V & $14.833 \pm 0.010$ \\
$2728.3823495-2728.3824653$ & R & $14.507 \pm 0.023$ \\
$2728.3827199-2728.3828935$ & B & $15.187 \pm 0.013$ \\
$2728.3832292-2728.3833449$ & V & $14.833 \pm 0.009$ \\
$2728.3835995-2728.3837153$ & R & $14.513 \pm 0.023$ \\
$2728.3839583-2728.3841319$ & B & $15.195 \pm 0.013$ \\
$2728.3848032-2728.3849190$ & R & $14.514 \pm 0.023$ \\
$2728.3853125-2728.3854282$ & B & $15.193 \pm 0.014$ \\
$2728.3857639-2728.3858796$ & V & $14.844 \pm 0.009$ \\
$2728.3861227-2728.3862384$ & R & $14.526 \pm 0.023$ \\
$2728.3875463-2728.3876620$ & V & $14.848 \pm 0.009$ \\
$2728.3880671-2728.3881829$ & R & $14.526 \pm 0.023$ \\
$2728.3890162-2728.3891898$ & B & $15.202 \pm 0.013$ \\
$2728.3894676-2728.3895833$ & V & $14.849 \pm 0.009$ \\
$2728.3898727-2728.3899884$ & R & $14.536 \pm 0.023$ \\
$2728.3904630-2728.3906366$ & B & $15.212 \pm 0.013$ \\
$2728.3909144-2728.3910301$ & V & $14.857 \pm 0.009$ \\
$2728.3913079-2728.3914236$ & R & $14.535 \pm 0.023$ \\
$2728.3917361-2728.3919097$ & B & $15.203 \pm 0.013$ \\
$2728.3921875-2728.3923032$ & V & $14.869 \pm 0.009$ \\
$2728.3925926-2728.3927083$ & R & $14.535 \pm 0.023$ \\
$2728.3930208-2728.3931944$ & B & $15.227 \pm 0.013$ \\
$2728.3934606-2728.3935764$ & V & $14.865 \pm 0.010$ \\
$2728.3938657-2728.3939815$ & R & $14.545 \pm 0.023$ \\
$2728.3942593-2728.3944329$ & B & $15.219 \pm 0.013$
\end{tabular}


Table 1-Continued

\begin{tabular}{ccc}
\hline \hline MJD & Band & Magnitude \\
\hline $2728.3947222-2728.3948380$ & V & $14.872 \pm 0.009$ \\
$2728.3951273-2728.3952431$ & R & $14.551 \pm 0.023$ \\
$2728.3956134-2728.3957870$ & B & $15.223 \pm 0.013$ \\
$2728.3961343-2728.3962500$ & V & $14.875 \pm 0.009$ \\
$2728.3965394-2728.3966551$ & R & $14.548 \pm 0.023$ \\
$2728.3969329-2728.3971065$ & B & $15.226 \pm 0.013$ \\
$2728.3973843-2728.3975000$ & V & $14.874 \pm 0.009$ \\
$2728.3977778-2728.3978935$ & R & $14.556 \pm 0.023$ \\
$2728.3983333-2728.3985069$ & B & $15.230 \pm 0.013$ \\
$2728.3988310-2728.3989468$ & V & $14.885 \pm 0.009$ \\
$2728.3992245-2728.3993403$ & R & $14.559 \pm 0.023$ \\
$2728.3996644-2728.3998380$ & B & $15.239 \pm 0.013$ \\
$2728.4001736-2728.4002894$ & V & $14.892 \pm 0.009$ \\
$2728.4006134-2728.4007292$ & R & $14.561 \pm 0.023$ \\
$2728.4010301-2728.4012037$ & B & $15.236 \pm 0.013$ \\
$2728.4014931-2728.4016088$ & V & $14.891 \pm 0.009$ \\
$2728.4019097-2728.4020255$ & R & $14.566 \pm 0.023$ \\
$2728.4023032-2728.4024769$ & B & $15.242 \pm 0.013$ \\
$2728.4027662-2728.4028819$ & V & $14.887 \pm 0.009$ \\
$2728.4031829-2728.4032986$ & R & $14.570 \pm 0.023$ \\
$2728.4036227-2728.4037963$ & B & $15.243 \pm 0.013$ \\
$2728.4040856-2728.4042014$ & V & $14.900 \pm 0.009$ \\
$2728.4050000-2728.4051157$ & R & $14.570 \pm 0.023$ \\
$2728.4054167-2728.4055903$ & B & $15.254 \pm 0.013$ \\
$2728.4059144-2728.4060301$ & V & $14.907 \pm 0.009$ \\
$2728.4063310-2728.4064468$ & R & $14.583 \pm 0.023$ \\
$2728.4068171-2728.4069907$ & B & $15.256 \pm 0.014$ \\
$2728.4072801-2728.4073958$ & V & $14.898 \pm 0.011$ \\
$2728.4076736-2728.4077894$ & R & $14.575 \pm 0.023$ \\
$2728.4082176-2728.4083912$ & B & $15.255 \pm 0.013$ \\
$2728.4086690-2728.4087847$ & V & $14.910 \pm 0.009$
\end{tabular}


Table 1-Continued

\begin{tabular}{ccc}
\hline \hline MJD & Band & Magnitude \\
\hline $2728.4090741-2728.4091898$ & R & $14.585 \pm 0.023$ \\
$2728.4094676-2728.4096412$ & B & $15.251 \pm 0.013$ \\
$2728.4099306-2728.4100463$ & V & $14.907 \pm 0.009$ \\
$2728.4103241-2728.4104398$ & R & $14.592 \pm 0.023$ \\
$2728.4107292-2728.4109028$ & B & $15.263 \pm 0.013$ \\
$2728.4111921-2728.4113079$ & V & $14.914 \pm 0.009$ \\
$2728.4116667-2728.4117824$ & R & $14.591 \pm 0.023$ \\
$2728.4120949-2728.4122685$ & B & $15.259 \pm 0.013$ \\
$2728.4126273-2728.4127431$ & V & $14.921 \pm 0.011$ \\
$2728.4130208-2728.4131366$ & R & $14.598 \pm 0.023$ \\
$2728.4134375-2728.4136111$ & B & $15.265 \pm 0.013$ \\
$2728.4138773-2728.4139931$ & V & $14.925 \pm 0.009$ \\
$2728.4143634-2728.4144792$ & R & $14.600 \pm 0.023$ \\
$2728.4147685-2728.4149421$ & B & $15.275 \pm 0.013$ \\
$2728.4154167-2728.4155324$ & V & $14.926 \pm 0.009$ \\
$2728.4160764-2728.4161921$ & R & $14.607 \pm 0.023$ \\
$2728.4165741-2728.4167477$ & B & $15.287 \pm 0.013$ \\
$2728.4170718-2728.4171875$ & V & $14.927 \pm 0.009$ \\
$2728.4177083-2728.4178241$ & R & $14.616 \pm 0.022$ \\
$2728.4182755-2728.4184491$ & B & $15.291 \pm 0.013$ \\
$2728.4188310-2728.4189468$ & V & $14.939 \pm 0.009$ \\
$2728.4193750-2728.4194907$ & R & $14.616 \pm 0.023$ \\
$2728.4198264-2728.4200000$ & B & $15.293 \pm 0.013$ \\
$2728.4202778-2728.4203935$ & V & $14.939 \pm 0.009$ \\
$2728.4207523-2728.4208681$ & R & $14.627 \pm 0.023$ \\
$2728.4211458-2728.4213194$ & B & $15.293 \pm 0.013$ \\
$2728.4216782-2728.4217940$ & V & $14.945 \pm 0.009$ \\
$2728.4220833-2728.4221991$ & R & $14.620 \pm 0.022$ \\
$2728.4226389-2728.4228125$ & B & $15.295 \pm 0.013$ \\
$2728.4230903-2728.4232060$ & V & $14.951 \pm 0.009$ \\
$2728.4234722-2728.4235880$ & R & $14.626 \pm 0.023$
\end{tabular}


Table 1-Continued

\begin{tabular}{ccc}
\hline \hline MJD & Band & Magnitude \\
\hline $2728.4238657-2728.4240394$ & B & $15.307 \pm 0.013$ \\
$2728.4243403-2728.4244560$ & V & $14.951 \pm 0.009$ \\
$2728.4247801-2728.4248958$ & R & $14.628 \pm 0.023$ \\
$2728.4252083-2728.4253819$ & B & $15.310 \pm 0.013$ \\
$2728.4256713-2728.4257870$ & V & $14.962 \pm 0.009$ \\
$2728.4260764-2728.4261921$ & R & $14.629 \pm 0.023$ \\
$2728.4264815-2728.4266551$ & B & $15.306 \pm 0.013$ \\
$2728.4269213-2728.4270370$ & V & $14.963 \pm 0.009$ \\
$2728.4273148-2728.4274306$ & R & $14.640 \pm 0.023$ \\
$2728.4277199-2728.4278935$ & B & $15.311 \pm 0.013$ \\
$2728.4281944-2728.4283102$ & V & $14.964 \pm 0.009$ \\
$2728.4285764-2728.4286921$ & R & $14.641 \pm 0.023$ \\
$2728.4291667-2728.4293403$ & B & $15.315 \pm 0.013$ \\
$2728.4296181-2728.4297338$ & V & $14.967 \pm 0.009$ \\
$2728.4300347-2728.4301505$ & R & $14.643 \pm 0.023$ \\
$2728.4304977-2728.4306713$ & B & $15.316 \pm 0.013$ \\
$2728.4309375-2728.4310532$ & V & $14.970 \pm 0.009$ \\
$2728.4313310-2728.4314468$ & R & $14.652 \pm 0.023$ \\
$2728.4317245-2728.4318981$ & B & $15.320 \pm 0.013$ \\
$2728.4321644-2728.4322801$ & V & $14.973 \pm 0.009$ \\
$2728.4326042-2728.4327199$ & R & $14.652 \pm 0.023$ \\
$2728.4329977-2728.4331713$ & B & $15.328 \pm 0.013$ \\
$2728.4341898-2728.4343056$ & V & $14.986 \pm 0.009$ \\
$2728.4345949-2728.4347106$ & R & $14.654 \pm 0.023$ \\
$2728.4350116-2728.4351852$ & B & $15.335 \pm 0.013$ \\
$2728.4354630-2728.4355787$ & V & $14.981 \pm 0.009$ \\
$2728.4358449-2728.4359606$ & R & $14.666 \pm 0.023$ \\
$2728.4362731-2728.4364468$ & B & $15.340 \pm 0.013$ \\
$2728.4367130-2728.4368287$ & V & $14.994 \pm 0.009$ \\
$2728.4370949-2728.4372106$ & R & $14.666 \pm 0.023$ \\
$2728.4375347-2728.4377083$ & B & $15.341 \pm 0.013$
\end{tabular}


Table 1-Continued

\begin{tabular}{ccc}
\hline \hline MJD & Band & Magnitude \\
\hline $2728.4379745-2728.4380903$ & V & $14.995 \pm 0.009$ \\
$2728.4383681-2728.4384838$ & R & $14.671 \pm 0.023$ \\
$2728.4387616-2728.4389352$ & B & $15.343 \pm 0.013$ \\
$2728.4392130-2728.4393287$ & V & $14.998 \pm 0.009$ \\
$2728.4396065-2728.4397222$ & R & $14.680 \pm 0.022$ \\
$2728.4399884-2728.4401620$ & B & $15.354 \pm 0.013$ \\
$2728.4404745-2728.4405903$ & V & $15.004 \pm 0.009$ \\
$2728.4409491-2728.4410648$ & R & $14.677 \pm 0.023$ \\
$2728.4413657-2728.4415394$ & B & $15.352 \pm 0.013$ \\
$2728.4418171-2728.4419329$ & V & $15.003 \pm 0.009$ \\
$2728.4422106-2728.4423264$ & R & $14.682 \pm 0.022$ \\
$2728.4426042-2728.4427778$ & B & $15.359 \pm 0.013$ \\
$2728.4430787-2728.4431944$ & V & $15.005 \pm 0.009$ \\
$2728.4434838-2728.4435995$ & R & $14.684 \pm 0.023$ \\
$2728.4438773-2728.4440509$ & B & $15.360 \pm 0.013$ \\
$2728.4443519-2728.4444676$ & V & $15.011 \pm 0.009$ \\
$2728.4447917-2728.4449074$ & R & $14.691 \pm 0.022$ \\
$2728.4452199-2728.4453935$ & B & $15.362 \pm 0.013$ \\
$2728.4456597-2728.4457755$ & V & $15.017 \pm 0.009$ \\
$2728.4460532-2728.4461690$ & R & $14.699 \pm 0.023$ \\
$2728.4464699-2728.4466435$ & B & $15.372 \pm 0.013$ \\
$2728.4469213-2728.4470370$ & V & $15.017 \pm 0.009$ \\
$2728.4473032-2728.4474190$ & R & $14.695 \pm 0.023$ \\
$2728.4476968-2728.4478704$ & B & $15.373 \pm 0.013$ \\
$2728.4481713-2728.4482870$ & V & $15.029 \pm 0.009$ \\
$2728.4485764-2728.4486921$ & R & $14.707 \pm 0.023$ \\
$2728.4490278-2728.4492014$ & B & $15.373 \pm 0.013$ \\
$2728.4495139-2728.4496296$ & V & $15.029 \pm 0.009$ \\
$2728.4499769-2728.4500926$ & R & $14.713 \pm 0.023$ \\
$2728.4503704-2728.4505440$ & B & $15.384 \pm 0.013$ \\
$2728.4508796-2728.4509954$ & V & $15.026 \pm 0.009$
\end{tabular}


Table 1-Continued

\begin{tabular}{ccc}
\hline \hline MJD & Band & Magnitude \\
\hline $2728.4513542-2728.4514699$ & $\mathrm{R}$ & $14.705 \pm 0.022$ \\
$2728.4517708-2728.4519444$ & $\mathrm{~B}$ & $15.381 \pm 0.013$ \\
$2728.4522222-2728.4523380$ & $\mathrm{~V}$ & $15.032 \pm 0.009$ \\
$2728.4527894-2728.4529051$ & $\mathrm{~V}$ & $15.037 \pm 0.009$ \\
$2728.4537269-2728.4538426$ & $\mathrm{R}$ & $14.716 \pm 0.023$ \\
$2728.4541204-2728.4542940$ & $\mathrm{~B}$ & $15.385 \pm 0.013$ \\
$2728.4545949-2728.4547106$ & $\mathrm{~V}$ & $15.041 \pm 0.009$ \\
$2728.4550347-2728.4551505$ & $\mathrm{R}$ & $14.719 \pm 0.023$ \\
$2728.4554167-2728.4555903$ & $\mathrm{~B}$ & $15.403 \pm 0.013$ \\
$2728.4559028-2728.4560185$ & $\mathrm{~V}$ & $15.050 \pm 0.009$ \\
$2728.4563194-2728.4564352$ & $\mathrm{R}$ & $14.720 \pm 0.022$ \\
$2728.4568056-2728.4569792$ & $\mathrm{~B}$ & $15.399 \pm 0.013$ \\
$2728.4572685-2728.4573843$ & $\mathrm{~V}$ & $15.048 \pm 0.009$ \\
$2728.4578356-2728.4579514$ & $\mathrm{R}$ & $14.724 \pm 0.022$ \\
$2728.4582639-2728.4584375$ & $\mathrm{~B}$ & $15.410 \pm 0.013$ \\
$2728.4587153-2728.4588310$ & $\mathrm{~V}$ & $15.052 \pm 0.009$ \\
$2728.4591435-2728.4592593$ & $\mathrm{R}$ & $14.729 \pm 0.022$ \\
$2728.4595602-2728.4597338$ & $\mathrm{~B}$ & $15.405 \pm 0.013$ \\
$2728.4601620-2728.4602778$ & $\mathrm{~V}$ & $15.059 \pm 0.009$ \\
$2728.4605556-2728.4606713$ & $\mathrm{R}$ & $14.734 \pm 0.022$ \\
$2728.4609954-2728.4611690$ & $\mathrm{~B}$ & $15.411 \pm 0.013$ \\
$2728.4614468-2728.4615625$ & $\mathrm{~V}$ & $15.061 \pm 0.009$ \\
$2728.4618866-2728.4620023$ & $\mathrm{R}$ & $14.738 \pm 0.023$ \\
$2728.4623148-2728.4624884$ & $\mathrm{~B}$ & $15.415 \pm 0.013$ \\
$2728.4627662-2728.4628819$ & $\mathrm{~V}$ & $15.071 \pm 0.009$ \\
$2728.4632292-2728.4633449$ & $\mathrm{R}$ & $14.749 \pm 0.023$ \\
$2728.4636574-2728.4638310$ & $\mathrm{~B}$ & $15.419 \pm 0.013$ \\
$2728.4641088-2728.4642245$ & $\mathrm{~V}$ & $15.067 \pm 0.009$ \\
$2728.4644907-2728.4646065$ & $\mathrm{R}$ & $14.745 \pm 0.023$ \\
$2728.4649306-2728.4651042$ & $\mathrm{~B}$ & $15.421 \pm 0.013$ \\
$2728.4653935-2728.4655093$ & $\mathrm{~V}$ & $15.078 \pm 0.009$
\end{tabular}


Table 1-Continued

\begin{tabular}{ccc}
\hline \hline MJD & Band & Magnitude \\
\hline $2728.4657755-2728.4658912$ & R & $14.753 \pm 0.023$ \\
$2728.4661806-2728.4663542$ & B & $15.427 \pm 0.013$ \\
$2728.4666551-2728.4667708$ & V & $15.079 \pm 0.009$ \\
$2728.4670255-2728.4671412$ & R & $14.751 \pm 0.023$ \\
$2728.4674421-2728.4676157$ & B & $15.428 \pm 0.013$ \\
$2728.4678704-2728.4679861$ & V & $15.081 \pm 0.009$ \\
$2728.4682870-2728.4684028$ & R & $14.757 \pm 0.022$ \\
$2728.4686921-2728.4688657$ & B & $15.437 \pm 0.013$ \\
$2728.4691667-2728.4692824$ & V & $15.087 \pm 0.009$ \\
$2728.4695949-2728.4697106$ & R & $14.759 \pm 0.023$ \\
$2728.4700463-2728.4702199$ & B & $15.435 \pm 0.013$ \\
$2728.4704861-2728.4706019$ & V & $15.088 \pm 0.009$ \\
$2728.4708796-2728.4709954$ & R & $14.764 \pm 0.023$ \\
$2728.4712847-2728.4714583$ & B & $15.443 \pm 0.013$ \\
$2728.4717477-2728.4718634$ & V & $15.092 \pm 0.010$ \\
$2728.4721412-2728.4722569$ & R & $14.770 \pm 0.023$ \\
$2728.4726273-2728.4728009$ & B & $15.454 \pm 0.013$ \\
$2728.4733449-2728.4734606$ & V & $15.099 \pm 0.009$ \\
$2728.4737269-2728.4738426$ & R & $14.771 \pm 0.022$ \\
$2728.4742477-2728.4744213$ & B & $15.454 \pm 0.013$ \\
$2728.4749074-2728.4750231$ & V & $15.103 \pm 0.009$ \\
$2728.4753125-2728.4754282$ & R & $14.779 \pm 0.023$ \\
$2728.4757060-2728.4758796$ & B & $15.454 \pm 0.013$ \\
$2728.4761458-2728.4762616$ & V & $15.104 \pm 0.009$ \\
$2728.4769444-2728.4771181$ & B & $15.465 \pm 0.013$ \\
$2728.4774074-2728.4775231$ & V & $15.117 \pm 0.009$ \\
$2728.4778125-2728.4779282$ & R & $14.784 \pm 0.022$ \\
$2728.4781944-2728.4783681$ & B & $15.466 \pm 0.013$ \\
$2728.4786343-2728.4787500$ & V & $15.115 \pm 0.009$ \\
$2728.4790509-2728.4791667$ & R & $14.790 \pm 0.023$ \\
$2728.4794444-2728.4796181$ & B & $15.468 \pm 0.013$
\end{tabular}


Table 1-Continued

\begin{tabular}{ccc}
\hline \hline MJD & Band & Magnitude \\
\hline $2728.4798958-2728.4800116$ & V & $15.116 \pm 0.009$ \\
$2728.4802778-2728.4803935$ & R & $14.801 \pm 0.023$ \\
$2728.4806713-2728.4808449$ & B & $15.460 \pm 0.014$ \\
$2728.4811227-2728.4812384$ & V & $15.121 \pm 0.009$ \\
$2728.4815162-2728.4816319$ & R & $14.802 \pm 0.023$ \\
$2728.4819097-2728.4820833$ & B & $15.477 \pm 0.013$ \\
$2728.4823958-2728.4825116$ & V & $15.130 \pm 0.010$ \\
$2728.4828819-2728.4829977$ & R & $14.809 \pm 0.023$ \\
$2728.4834259-2728.4835995$ & B & $15.482 \pm 0.014$ \\
$2728.4839815-2728.4840972$ & V & $15.141 \pm 0.010$ \\
$2728.4843866-2728.4845023$ & R & $14.808 \pm 0.023$ \\
$2728.4847801-2728.4849537$ & B & $15.489 \pm 0.014$ \\
$2728.4852546-2728.4853704$ & V & $15.140 \pm 0.011$ \\
$2728.4856829-2728.4857986$ & R & $14.815 \pm 0.024$ \\
$2728.4862500-2728.4864236$ & B & $15.495 \pm 0.014$ \\
$2728.4867014-2728.4868171$ & V & $15.150 \pm 0.012$ \\
$2728.4871296-2728.4872454$ & R & $14.810 \pm 0.024$ \\
$2728.4875347-2728.4877083$ & B & $15.503 \pm 0.014$ \\
$2728.4880093-2728.4881250$ & V & $15.149 \pm 0.011$ \\
$2728.4883912-2728.4885069$ & R & $14.827 \pm 0.023$ \\
$2728.4887847-2728.4889583$ & B & $15.488 \pm 0.014$ \\
$2728.4892361-2728.4893519$ & V & $15.150 \pm 0.010$ \\
$2728.4896644-2728.4897801$ & R & $14.827 \pm 0.023$ \\
$2728.4901389-2728.4903125$ & B & $15.502 \pm 0.014$ \\
$2728.4905903-2728.4907060$ & V & $15.153 \pm 0.011$ \\
$2728.4909954-2728.4911111$ & R & $14.833 \pm 0.024$ \\
$2728.4914120-2728.4915856$ & B & $15.502 \pm 0.014$ \\
$2728.4918519-2728.4919676$ & V & $15.156 \pm 0.010$ \\
$2728.4922454-2728.4923611$ & R & $14.832 \pm 0.024$ \\
$2728.4926505-2728.4928241$ & B & $15.511 \pm 0.013$ \\
$2728.4931366-2728.4932523$ & V & $15.168 \pm 0.012$
\end{tabular}


Table 1-Continued

\begin{tabular}{ccc}
\hline \hline MJD & Band & Magnitude \\
\hline $2728.4935764-2728.4936921$ & $\mathrm{R}$ & $14.833 \pm 0.023$ \\
$2728.4940162-2728.4941898$ & $\mathrm{~B}$ & $15.517 \pm 0.014$ \\
$2728.4944792-2728.4945949$ & $\mathrm{~V}$ & $15.166 \pm 0.010$ \\
$2728.4948727-2728.4949884$ & $\mathrm{R}$ & $14.840 \pm 0.023$ \\
$2728.4952894-2728.4954630$ & $\mathrm{~B}$ & $15.516 \pm 0.016$ \\
$2728.4957523-2728.4958681$ & $\mathrm{~V}$ & $15.173 \pm 0.013$ \\
$2728.4961343-2728.4962500$ & $\mathrm{R}$ & $14.847 \pm 0.025$ \\
$2728.4965509-2728.4967245$ & $\mathrm{~B}$ & $15.522 \pm 0.014$ \\
$2728.4969907-2728.4971065$ & $\mathrm{~V}$ & $15.172 \pm 0.012$ \\
$2728.4973958-2728.4975116$ & $\mathrm{R}$ & $14.846 \pm 0.027$ \\
$2728.4978241-2728.4979977$ & $\mathrm{~B}$ & $15.525 \pm 0.018$ \\
$2728.4983218-2728.4984375$ & $\mathrm{~V}$ & $15.173 \pm 0.013$ \\
$2728.4987963-2728.4989120$ & $\mathrm{R}$ & $14.857 \pm 0.024$ \\
$2728.4991898-2728.4993634$ & $\mathrm{~B}$ & $15.520 \pm 0.014$ \\
$2728.4996759-2728.4997917$ & $\mathrm{~V}$ & $15.181 \pm 0.013$ \\
$2728.5000810-2728.5001968$ & $\mathrm{R}$ & $14.848 \pm 0.026$ \\
$2728.5005787-2728.5007523$ & $\mathrm{~B}$ & $15.541 \pm 0.015$ \\
$2728.5010185-2728.5011343$ & $\mathrm{~V}$ & $15.184 \pm 0.014$ \\
$2728.5014236-2728.5015394$ & $\mathrm{R}$ & $14.859 \pm 0.027$ \\
$2728.5018056-2728.5019792$ & $\mathrm{~B}$ & $15.530 \pm 0.015$ \\
$2728.5022685-2728.5023843$ & $\mathrm{~V}$ & $15.182 \pm 0.010$ \\
$2728.5026736-2728.5027894$ & $\mathrm{R}$ & $14.866 \pm 0.023$ \\
$2728.5030903-2728.5032639$ & $\mathrm{~B}$ & $15.534 \pm 0.014$ \\
$2728.5035301-2728.5036458$ & $\mathrm{~V}$ & $15.199 \pm 0.010$ \\
$2728.5039815-2728.5040972$ & $\mathrm{R}$ & $14.870 \pm 0.023$ \\
$2728.5044329-2728.5046065$ & $\mathrm{~B}$ & $15.546 \pm 0.013$ \\
$2728.5048958-2728.5050116$ & $\mathrm{~V}$ & $15.202 \pm 0.010$ \\
$2728.5052778-2728.5053935$ & $\mathrm{R}$ & $14.867 \pm 0.023$ \\
$2728.5056713-2728.5058449$ & $\mathrm{~B}$ & $15.540 \pm 0.014$ \\
$2728.5061111-2728.5062269$ & $\mathrm{~V}$ & $15.198 \pm 0.010$ \\
$2728.5067014-2728.5068171$ & $\mathrm{R}$ & $14.878 \pm 0.023$
\end{tabular}


Table 1-Continued

\begin{tabular}{ccc}
\hline \hline MJD & Band & Magnitude \\
\hline $2728.5075231-2728.5076968$ & B & $15.552 \pm 0.013$ \\
$2728.5081944-2728.5083102$ & V & $15.205 \pm 0.010$ \\
$2728.5086921-2728.5088079$ & R & $14.879 \pm 0.023$ \\
$2728.5091319-2728.5093056$ & B & $15.560 \pm 0.014$ \\
$2728.5096991-2728.5098148$ & V & $15.206 \pm 0.010$ \\
$2728.5101389-2728.5102546$ & R & $14.889 \pm 0.025$ \\
$2728.5105440-2728.5107176$ & B & $15.547 \pm 0.014$ \\
$2728.5110069-2728.5111227$ & V & $15.221 \pm 0.013$ \\
$2728.5116898-2728.5118056$ & R & $14.896 \pm 0.026$ \\
$2728.5121296-2728.5123032$ & B & $15.565 \pm 0.014$ \\
$2728.5127894-2728.5129051$ & V & $15.219 \pm 0.012$ \\
$2728.5132407-2728.5133565$ & R & $14.902 \pm 0.024$ \\
$2728.5138079-2728.5139815$ & B & $15.566 \pm 0.014$ \\
$2728.5142824-2728.5143981$ & V & $15.218 \pm 0.011$ \\
$2728.5147106-2728.5148264$ & R & $14.894 \pm 0.026$ \\
$2728.5152315-2728.5154051$ & B & $15.570 \pm 0.015$ \\
$2728.5156944-2728.5158102$ & V & $15.219 \pm 0.012$ \\
$2728.5163542-2728.5164699$ & R & $14.910 \pm 0.023$ \\
$2728.5167708-2728.5169444$ & B & $15.583 \pm 0.014$ \\
$2728.5172106-2728.5173264$ & V & $15.228 \pm 0.011$ \\
$2728.5176273-2728.5177431$ & R & $14.914 \pm 0.025$ \\
$2728.5180903-2728.5182639$ & B & $15.592 \pm 0.015$ \\
$2728.5185995-2728.5187153$ & V & $15.236 \pm 0.013$ \\
$2728.5191088-2728.5192245$ & R & $14.918 \pm 0.024$ \\
$2728.5197106-2728.5198843$ & B & $15.590 \pm 0.021$ \\
$2728.5201736-2728.5202894$ & V & $15.252 \pm 0.016$ \\
$2728.5206829-2728.5207986$ & R & $14.915 \pm 0.035$ \\
$2728.5212269-2728.5214005$ & B & $15.597 \pm 0.021$ \\
$2728.5216667-2728.5217824$ & V & $15.242 \pm 0.023$ \\
$2728.5220718-2728.5221875$ & R & $14.922 \pm 0.068$ \\
$2728.5235995-2728.5238310$ & V & $15.254 \pm 0.021$
\end{tabular}


Table 1-Continued

\begin{tabular}{ccc}
\hline \hline MJD & Band & Magnitude \\
\hline $2728.5242824-2728.5246296$ & R & $14.933 \pm 0.026$ \\
$2728.5251389-2728.5256597$ & B & $15.604 \pm 0.014$ \\
$2728.5260069-2728.5263542$ & V & $15.262 \pm 0.009$ \\
$2728.5274190-2728.5277662$ & R & $14.933 \pm 0.027$ \\
$2728.5284491-2728.5289699$ & B & $15.618 \pm 0.013$ \\
$2728.5293056-2728.5296528$ & V & $15.264 \pm 0.009$ \\
$2728.5300231-2728.5303704$ & R & $14.947 \pm 0.023$ \\
$2728.5307639-2728.5312847$ & B & $15.612 \pm 0.014$ \\
$2728.5317245-2728.5320718$ & V & $15.270 \pm 0.010$ \\
$2728.5327546-2728.5329861$ & R & $14.954 \pm 0.025$ \\
$2728.5335995-2728.5339468$ & B & $15.619 \pm 0.032$ \\
$2728.5342477-2728.5344792$ & V & $15.270 \pm 0.015$ \\
$2728.5347917-2728.5350231$ & R & $14.956 \pm 0.024$ \\
$2728.5355093-2728.5358565$ & B & $15.626 \pm 0.013$ \\
$2728.5362616-2728.5364931$ & V & $15.281 \pm 0.010$ \\
$2728.5369444-2728.5371759$ & R & $14.961 \pm 0.023$ \\
$2728.5375347-2728.5378819$ & B & $15.630 \pm 0.014$ \\
$2728.5381481-2728.5383796$ & V & $15.290 \pm 0.011$ \\
$2728.5388079-2728.5390394$ & R & $14.972 \pm 0.023$ \\
$2728.5393866-2728.5397338$ & B & $15.635 \pm 0.013$ \\
$2728.5400926-2728.5403241$ & V & $15.289 \pm 0.010$ \\
$2728.5406366-2728.5408681$ & R & $14.977 \pm 0.026$ \\
$2728.5411343-2728.5414815$ & B & $15.644 \pm 0.016$ \\
$2728.5418056-2728.5420370$ & V & $15.306 \pm 0.012$ \\
$2728.5424421-2728.5426736$ & R & $14.979 \pm 0.024$ \\
$2728.5429977-2728.5433449$ & B & $15.648 \pm 0.013$ \\
$2728.5439120-2728.5441435$ & V & $15.313 \pm 0.010$ \\
$2728.5444213-2728.5446528$ & R & $14.984 \pm 0.023$ \\
$2728.5453704-2728.5456019$ & V & $15.303 \pm 0.009$ \\
$2728.5459144-2728.5461458$ & R & $14.993 \pm 0.023$ \\
$2728.5464352-2728.5467824$ & B & $15.654 \pm 0.014$
\end{tabular}


Table 1-Continued

\begin{tabular}{ccc}
\hline \hline MJD & Band & Magnitude \\
\hline $2728.5470718-2728.5473032$ & V & $15.314 \pm 0.010$ \\
$2728.5476852-2728.5479167$ & R & $14.992 \pm 0.023$ \\
$2728.5482176-2728.5485648$ & B & $15.665 \pm 0.013$ \\
$2728.5488426-2728.5490741$ & V & $15.326 \pm 0.010$ \\
$2728.5493750-2728.5496065$ & R & $14.995 \pm 0.023$ \\
$2728.5499074-2728.5502546$ & B & $15.676 \pm 0.013$ \\
$2728.5506366-2728.5508681$ & V & $15.320 \pm 0.010$ \\
$2728.5512269-2728.5514583$ & R & $15.003 \pm 0.025$ \\
$2728.5571644-2728.5575116$ & B & $15.691 \pm 0.017$ \\
$2728.5578819-2728.5581134$ & V & $15.339 \pm 0.014$ \\
$2728.5586458-2728.5588773$ & R & $15.027 \pm 0.029$ \\
$2728.5591782-2728.5595255$ & B & $15.699 \pm 0.026$ \\
$2728.5598032-2728.5600347$ & V & $15.357 \pm 0.027$ \\
$2728.5603241-2728.5605556$ & R & $15.031 \pm 0.025$ \\
$2728.5608565-2728.5612037$ & B & $15.713 \pm 0.014$ \\
$2728.5617245-2728.5619560$ & V & $15.365 \pm 0.010$ \\
$2728.5622222-2728.5624537$ & R & $15.036 \pm 0.023$ \\
$2728.5627315-2728.5630787$ & B & $15.709 \pm 0.014$ \\
$2728.5633681-2728.5635995$ & V & $15.371 \pm 0.012$ \\
$2728.5639005-2728.5641319$ & R & $15.042 \pm 0.024$ \\
$2728.5644213-2728.5647685$ & B & $15.718 \pm 0.014$ \\
$2728.5650463-2728.5652778$ & V & $15.372 \pm 0.010$ \\
$2728.5655671-2728.5657986$ & R & $15.042 \pm 0.024$ \\
$2728.5663079-2728.5666551$ & B & $15.717 \pm 0.014$ \\
$2728.5669560-2728.5671875$ & V & $15.365 \pm 0.012$ \\
$2728.5675810-2728.5678125$ & R & $15.055 \pm 0.036$ \\
$2728.5681597-2728.5685069$ & B & $15.731 \pm 0.014$ \\
$2728.5688657-2728.5690972$ & V & $15.373 \pm 0.013$ \\
$2728.5694444-2728.5696759$ & R & $15.055 \pm 0.024$ \\
$2728.5700810-2728.5704282$ & B & $15.739 \pm 0.014$ \\
$2728.5707176-2728.5709491$ & V & $15.373 \pm 0.014$
\end{tabular}


Table 1-Continued

\begin{tabular}{ccc}
\hline \hline MJD & Band & Magnitude \\
\hline $2728.5712269-2728.5714583$ & $\mathrm{R}$ & $15.063 \pm 0.024$ \\
$2728.5717130-2728.5720602$ & $\mathrm{~B}$ & $15.732 \pm 0.015$ \\
$2728.5723264-2728.5725579$ & $\mathrm{~V}$ & $15.405 \pm 0.024$ \\
$2728.5729051-2728.5730208$ & $\mathrm{R}$ & $15.069 \pm 0.026$ \\
$2728.5732986-2728.5735301$ & $\mathrm{R}$ & $15.073 \pm 0.024$ \\
$2728.5738773-2728.5742245$ & $\mathrm{~B}$ & $15.746 \pm 0.013$ \\
$2728.5745139-2728.5747454$ & $\mathrm{~V}$ & $15.401 \pm 0.011$ \\
$2728.5750694-2728.5753009$ & $\mathrm{R}$ & $15.083 \pm 0.024$ \\
$2728.5756134-2728.5759606$ & $\mathrm{~B}$ & $15.743 \pm 0.013$ \\
$2728.5762731-2728.5765046$ & $\mathrm{~V}$ & $15.399 \pm 0.009$ \\
$2728.5769560-2728.5771875$ & $\mathrm{R}$ & $15.080 \pm 0.023$ \\
$2728.5774884-2728.5778356$ & $\mathrm{~B}$ & $15.752 \pm 0.013$ \\
$2728.5781597-2728.5783912$ & $\mathrm{~V}$ & $15.411 \pm 0.009$ \\
$2728.5786690-2728.5789005$ & $\mathrm{R}$ & $15.081 \pm 0.023$ \\
$2728.5792014-2728.5795486$ & $\mathrm{~B}$ & $15.767 \pm 0.014$ \\
$2728.5798611-2728.5800926$ & $\mathrm{~V}$ & $15.406 \pm 0.011$ \\
$2728.5803819-2728.5806134$ & $\mathrm{R}$ & $15.092 \pm 0.023$ \\
$2728.5809491-2728.5812963$ & $\mathrm{~B}$ & $15.766 \pm 0.013$ \\
$2728.5817708-2728.5820023$ & $\mathrm{~V}$ & $15.414 \pm 0.009$ \\
$2728.5823148-2728.5825463$ & $\mathrm{R}$ & $15.100 \pm 0.023$ \\
$2728.5828704-2728.5832176$ & $\mathrm{~B}$ & $15.773 \pm 0.013$ \\
$2728.5834838-2728.5837153$ & $\mathrm{~V}$ & $15.423 \pm 0.009$ \\
$2728.5839699-2728.5842014$ & $\mathrm{R}$ & $15.101 \pm 0.022$ \\
$2728.5845833-2728.5849306$ & $\mathrm{~B}$ & $15.776 \pm 0.013$ \\
$2728.5852199-2728.5854514$ & $\mathrm{~V}$ & $15.430 \pm 0.009$ \\
$2728.5857292-2728.5859606$ & $\mathrm{R}$ & $15.109 \pm 0.022$ \\
$2728.5862616-2728.5866088$ & $\mathrm{~B}$ & $15.782 \pm 0.013$ \\
$2728.5869329-2728.5871644$ & $\mathrm{~V}$ & $15.432 \pm 0.009$ \\
$2728.5874421-2728.5876736$ & $\mathrm{R}$ & $15.111 \pm 0.022$ \\
$2728.5880208-2728.5883681$ & $\mathrm{~B}$ & $15.790 \pm 0.013$ \\
$2728.5886343-2728.5888657$ & $\mathrm{~V}$ & $15.440 \pm 0.009$
\end{tabular}


Table 1-Continued

\begin{tabular}{ccc}
\hline \hline MJD & Band & Magnitude \\
\hline $2728.5892245-2728.5894560$ & R & $15.122 \pm 0.023$ \\
$2728.5897685-2728.5901157$ & B & $15.794 \pm 0.014$ \\
$2728.5903935-2728.5906250$ & V & $15.439 \pm 0.012$ \\
$2728.5908912-2728.5911227$ & R & $15.120 \pm 0.023$ \\
$2728.5913773-2728.5917245$ & B & $15.807 \pm 0.014$ \\
$2728.5920718-2728.5923032$ & V & $15.463 \pm 0.010$ \\
$2728.5925694-2728.5928009$ & R & $15.120 \pm 0.024$ \\
$2728.5931134-2728.5934606$ & B & $15.810 \pm 0.014$ \\
$2728.5937847-2728.5940162$ & V & $15.461 \pm 0.010$ \\
$2728.5943519-2728.5945833$ & R & $15.130 \pm 0.024$ \\
$2728.5949537-2728.5953009$ & B & $15.810 \pm 0.014$ \\
$2728.5956481-2728.5958796$ & V & $15.469 \pm 0.010$ \\
$2728.5962384-2728.5964699$ & R & $15.132 \pm 0.024$ \\
$2728.5969213-2728.5972685$ & B & $15.812 \pm 0.014$ \\
$2728.5975231-2728.5977546$ & V & $15.469 \pm 0.010$ \\
$2728.5980671-2728.5982986$ & R & $15.135 \pm 0.023$ \\
$2728.5986111-2728.5989583$ & B & $15.817 \pm 0.014$ \\
$2728.5993056-2728.5995370$ & V & $15.457 \pm 0.011$ \\
$2728.5998032-2728.6000347$ & R & $15.158 \pm 0.024$ \\
$2728.6003819-2728.6007292$ & B & $15.828 \pm 0.014$ \\
$2728.6010417-2728.6012731$ & V & $15.467 \pm 0.010$ \\
$2728.6015278-2728.6017593$ & R & $15.159 \pm 0.023$ \\
$2728.6020718-2728.6024190$ & B & $15.836 \pm 0.013$ \\
$2728.6026852-2728.6029167$ & V & $15.481 \pm 0.009$ \\
$2728.6032176-2728.6034491$ & R & $15.163 \pm 0.022$ \\
$2728.6039931-2728.6043403$ & B & $15.838 \pm 0.013$ \\
$2728.6046644-2728.6048958$ & V & $15.482 \pm 0.009$ \\
$2728.6051736-2728.6054051$ & R & $15.161 \pm 0.022$ \\
$2728.6056713-2728.6060185$ & B & $15.841 \pm 0.013$ \\
$2728.6063310-2728.6065625$ & V & $15.493 \pm 0.010$ \\
$2728.6069560-2728.6071875$ & R & $15.169 \pm 0.023$
\end{tabular}


Table 1-Continued

\begin{tabular}{ccc}
\hline \hline MJD & Band & Magnitude \\
\hline $2728.6074884-2728.6078356$ & B & $15.850 \pm 0.013$ \\
$2728.6081134-2728.6083449$ & V & $15.483 \pm 0.011$ \\
$2728.6086343-2728.6088657$ & R & $15.184 \pm 0.024$ \\
$2728.6091435-2728.6094907$ & B & $15.849 \pm 0.014$ \\
$2728.6098148-2728.6100463$ & V & $15.513 \pm 0.010$ \\
$2728.6103935-2728.6106250$ & R & $15.186 \pm 0.023$ \\
$2728.6109144-2728.6112616$ & B & $15.858 \pm 0.013$ \\
$2728.6115509-2728.6117824$ & V & $15.508 \pm 0.010$ \\
$2728.6120486-2728.6122801$ & R & $15.187 \pm 0.023$ \\
$2728.6125810-2728.6129282$ & B & $15.861 \pm 0.013$ \\
$2728.6132292-2728.6134606$ & V & $15.503 \pm 0.010$ \\
$2728.6137384-2728.6139699$ & R & $15.197 \pm 0.023$ \\
$2728.6142940-2728.6146412$ & B & $15.872 \pm 0.013$ \\
$2728.6150116-2728.6152431$ & V & $15.509 \pm 0.010$ \\
$2728.6155556-2728.6157870$ & R & $15.187 \pm 0.023$ \\
$2728.6160995-2728.6164468$ & B & $15.876 \pm 0.013$ \\
$2728.6167477-2728.6169792$ & V & $15.515 \pm 0.009$ \\
$2728.6172569-2728.6174884$ & R & $15.191 \pm 0.023$ \\
$2728.6177778-2728.6181250$ & B & $15.886 \pm 0.013$ \\
$2728.6185648-2728.6187963$ & V & $15.529 \pm 0.010$ \\
$2728.6191667-2728.6193981$ & R & $15.211 \pm 0.023$ \\
$2728.6196875-2728.6200347$ & B & $15.892 \pm 0.013$ \\
$2728.6208796-2728.6211111$ & R & $15.205 \pm 0.023$ \\
$2728.6214931-2728.6218403$ & B & $15.897 \pm 0.013$ \\
$2728.6221644-2728.6223958$ & V & $15.548 \pm 0.014$ \\
$2728.6228009-2728.6230324$ & R & $15.208 \pm 0.023$ \\
$2728.6233449-2728.6236921$ & B & $15.908 \pm 0.014$ \\
$2728.6239931-2728.6242245$ & V & $15.541 \pm 0.010$ \\
$2728.6245370-2728.6247685$ & R & $15.226 \pm 0.023$ \\
$2728.6251505-2728.6254977$ & B & $15.912 \pm 0.014$ \\
$2728.6257986-2728.6260301$ & V & $15.549 \pm 0.011$
\end{tabular}


Table 1-Continued

\begin{tabular}{ccc}
\hline \hline MJD & Band & Magnitude \\
\hline $2728.6263194-2728.6265509$ & $\mathrm{R}$ & $15.228 \pm 0.024$ \\
$2728.6269792-2728.6273264$ & $\mathrm{~B}$ & $15.919 \pm 0.013$ \\
$2728.6277431-2728.6279745$ & $\mathrm{~V}$ & $15.559 \pm 0.010$ \\
$2728.6283681-2728.6285995$ & $\mathrm{R}$ & $15.227 \pm 0.023$ \\
$2728.6289583-2728.6293056$ & $\mathrm{~B}$ & $15.921 \pm 0.013$ \\
$2728.6296065-2728.6298380$ & $\mathrm{~V}$ & $15.563 \pm 0.011$ \\
$2728.6302778-2728.6305093$ & $\mathrm{R}$ & $15.236 \pm 0.024$ \\
$2728.6308681-2728.6312153$ & $\mathrm{~B}$ & $15.933 \pm 0.014$ \\
$2728.6315162-2728.6317477$ & $\mathrm{~V}$ & $15.567 \pm 0.010$ \\
$2728.6322569-2728.6324884$ & $\mathrm{R}$ & $15.239 \pm 0.024$ \\
\hline
\end{tabular}

